\title{
Impulsive control on delayed feedback chaotic financial system with Markovian jumping
}

\author{
Ruofeng Rao ${ }^{1,3^{*}}$ (D) and Shouming Zhong ${ }^{2,3}$
}

\author{
"Correspondence: \\ ruofengrao@163.com \\ 'Department of Mathematics, \\ Chengdu Normal University, \\ Chengdu, China \\ ${ }^{3}$ Institute of Financial Mathematics, \\ Chengdu Normal University, \\ Chengdu, China \\ Full list of author information is \\ available at the end of the article
}

\begin{abstract}
In this paper, the global asymptotic stability of both the closed economy system and the open economy system is investigated under impulse control, and the obtained stability criteria improve the existing results in the previous literature, generalizing the stabilization from the closed economy system to the open economy system, and stabilizing the unstable equilibrium point with positive interest rate. Particularly, stability of the equilibrium point with positive interest rate is suitable for the open economic market of China, for the interest rates during different periods in China's financial market are always some of positive percentages. Finally, numerical examples illustrate the effectiveness of the proposed methods.
\end{abstract}

Keywords: Positive interest rate; Chaotic financial system; Reduction to absurdity; Impulse control; Markovian jumping

\section{Introduction}

The dynamic behavior of a complex financial system is unpredictable and unstable. Delay is determined by the nature of delayed feedback in financial markets. Delay makes the dynamic behavior of a chaotic financial system more unpredictable. To avoid the economic crisis and financial risks, the government's macroeconomic control makes it necessary to study the stability of the financial system. Impulse control is one of the effective means for macroeconomic regulation and control of financial market. So we may firstly introduce a complex financial system. Establishing and testing a financial model consisting of production sub-blocks, currency sub-blocks, securities sub-blocks, and labor sub-blocks by using system dynamics method, people find that some long-term behaviors given by the model are irregular and extremely sensitive to the parameter changes of initial state values. The above-mentioned financial system is presented as follows:

$$
\left\{\begin{array}{l}
\dot{x}=z+(y-a) x, \\
\dot{y}=1-b y-x^{2}, \\
\dot{z}=-x-c z,
\end{array}\right.
$$

(c) The Author(s) 2020. This article is licensed under a Creative Commons Attribution 4.0 International License, which permits use sharing, adaptation, distribution and reproduction in any medium or format, as long as you give appropriate credit to the original author(s) and the source, provide a link to the Creative Commons licence, and indicate if changes were made. The images or other third party material in this article are included in the article's Creative Commons licence, unless indicated otherwise in a credit line to the material. If material is not included in the article's Creative Commons licence and your intended use is not permitted by statutory regulation or exceeds the permitted use, you will need to obtain permission directly from the copyright holder. To view a copy of this licence, visit http://creativecommons.org/licenses/by/4.0/. 
which has been investigated in many existing literature works [1-8], where $x$ represents the interest rate, $y$ represents the investment demand, $z$ represents the price index, $a$ represents savings, $b$ represents the unit investment cost, and $c$ represents the elasticity of commodity demand. Since people's behavior is rational, they have normal delayed response when facing market changes. So the delayed feedback system was introduced as follows $[5,6,8]$ :

$$
\left\{\begin{array}{l}
\dot{x}=z+(y-a) x+k_{1}\left(x-x\left(t-\tau_{1}(t)\right)\right) \\
\dot{y}=1-b y-x^{2}+k_{2}\left(y-y\left(t-\tau_{2}(t)\right)\right) \\
\dot{z}=-x-c z+k_{3}\left(z-z\left(t-\tau_{3}(t)\right)\right)
\end{array}\right.
$$

where $k_{i}(i=1,2,3)$ is the feedback gain coefficient.

In system (1.1), if $c-b-a b c \leq 0$, financial system (1.1) has the unique equilibrium point $Q_{0}\left(0, \frac{1}{b}, 0\right)$; if $c-b-a b c \geq 0$, financial system (1.1) owns three equilibrium points $Q_{0}\left(0, \frac{1}{b}, 0\right), Q_{1}\left(\sqrt{\theta}, \frac{1+a c}{c}, \frac{-\sqrt{\theta}}{c}\right), Q_{2}\left(-\sqrt{\theta}, \frac{1+a c}{c}, \frac{\sqrt{\theta}}{c}\right)$, where $\theta=\sqrt{\frac{c-b-a b c}{c}}$. Chaos appears in financial system (1.1) if $c-b-a b c>0$ and $c+a-\frac{1}{b}<0$. For example, let $a=0.9, b=0.2$, $c=1.2$, then there is a chaos phenomenon in financial system (1.1) (see, e.g., [8, Fig. 1]).

Moreover, time delays make the dynamic behavior of the financial system even more complex and unpredictable (see, e.g., [8, Fig. 2]). In fact, time delay brings essential difficulties to impulse control of a delayed feedback financial system. Existing stability criteria of the equilibrium point $Q_{1}\left(\sqrt{\theta}, \frac{1+a c}{c}, \frac{-\sqrt{\theta}}{c}\right)$ derived solely by impulse control in some literature are probably erroneous. For example, such literature is always involved in citing the erroneous conclusions of [9]. In fact, Yinping Zhang and Qing-Guo Wang in [10] pointed out the errors of [9, Lemma 3] and [9, Theorem 1]. So the author of [8] had to employ simultaneously impulse control and regional control on the delayed feedback system (see [8, Theorem 1]). Of course, impulse control is effective for the financial system without any time delays (see [8, Theorem 2]). In [11-20], global asymptotic stability of dynamics of a nonlinear dynamical system was discussed. Motivated by some methods of the related literature $[8,11-26]$, we shall discuss the global asymptotic stability of chaotic economy systems and employ solely impulse control on the financial system with small time delays. Besides, robust stability is very important to the nonlinear dynamic system [27], and hence we may consider the robust stability for the financial system with parameter uncertainties. By employing some mathematical analysis techniques and Lyapunov function methods, we shall give the globally exponential stability criteria for the closed economy system and the open economy system.

Remark 1 In the previous related literature, only the closed economy system was involved $[8,21-26,28]$. But in this paper, both the closed economy system and the open economy system are simultaneously investigated under impulse control.

\section{Preparation}

Firstly, we introduce the financial mathematical model investigated in this paper. 
Let

$$
\left\{\begin{array}{l}
X_{1}=x-\sqrt{\theta} \\
X_{2}=y-\frac{1+a c}{c} \\
X_{3}=z+\frac{\sqrt{\theta}}{c}
\end{array}\right.
$$

then the delayed feedback financial system (1.2) is translated into the following system:

$$
\left\{\begin{array}{l}
\dot{X}_{1}=\frac{1}{c} X_{1}+\theta X_{2}+X_{3}+X_{1} X_{2}+k_{1}\left(X_{1}-X_{1}\left(t-\tau_{1}(t)\right)\right) \\
\dot{X}_{2}=-2 \theta X_{1}-b X_{2}-X_{1}^{2}+k_{2}\left(X_{2}-X_{2}\left(t-\tau_{2}(t)\right)\right) \\
\dot{X}_{3}=-X_{1}-c X_{3}+k_{3}\left(X_{3}-X_{3}\left(t-\tau_{3}(t)\right)\right)
\end{array}\right.
$$

or

$$
\dot{X}(t)=-A X(t)+f(X(t))+K(X-X(t-\tau(t)))
$$

where the equilibrium point $Q_{1}\left(\sqrt{\theta}, \frac{1+a c}{c}, \frac{-\sqrt{\theta}}{c}\right)$ of the delayed feedback system (1.2) corresponds to the null solution of system (2.2) or (2.3), and $A, K$, and $f$ are defined as follows:

$$
A=\left(\begin{array}{ccc}
-\frac{1}{c} & -\theta & -1 \\
2 \theta & b & 0 \\
1 & 0 & c
\end{array}\right), \quad f(X)=\left(\begin{array}{c}
X_{1} X_{2} \\
-X_{1}^{2} \\
0
\end{array}\right), \quad K=\left(\begin{array}{ccc}
k_{1} & 0 & 0 \\
0 & k_{2} & 0 \\
0 & 0 & k_{3}
\end{array}\right)
$$

The so-called impulse control system is proposed as follows:

$$
\left\{\begin{array}{l}
\dot{X}(t)=-A X(t)+f(X(t))+K(X-X(t-\tau(t))), \quad t \geq t_{0}=0, t \neq t_{k}, k \in \mathbb{Z}^{+}, \\
X\left(t_{k}^{+}\right)=X\left(t_{k}\right)=B_{k} X\left(t_{k}^{-}\right), \quad t=t_{k}, k \in \mathbb{Z}^{+} \triangleq\{1,2, \ldots\}, \\
X(s)=\xi(s), \quad s \in[-\tau, 0]
\end{array}\right.
$$

or

$$
\left\{\begin{array}{l}
\dot{x}=z+(y-a) x+k_{1}\left(x-x\left(t-\tau_{1}(t)\right)\right), \quad t \geq 0, t \neq t_{k}, k=1,2, \ldots, \\
\dot{y}=1-b y-x^{2}+k_{2}\left(y-y\left(t-\tau_{2}(t)\right)\right), \quad t \geq 0, t \neq t_{k}, k=1,2, \ldots, \\
\dot{z}=-x-c z+k_{3}\left(z-z\left(t-\tau_{3}(t)\right)\right), \quad t \geq 0, t \neq t_{k}, k=1,2, \ldots, \\
\left(x\left(t_{k}^{+}\right)-\sqrt{\theta}, y\left(t_{k}^{+}\right)-\frac{1+a c}{c}, z\left(t_{k}^{+}\right)+\frac{\sqrt{\theta}}{c}\right)^{T} \\
\quad=B_{k}\left(x\left(t_{k}^{-}\right)-\sqrt{\theta}, y\left(t_{k}^{-}\right)-\frac{1+a c}{c}, z\left(t_{k}^{-}\right)+\frac{\sqrt{\theta}}{c}\right)^{T}, \quad t=t_{k}, k \in \mathbb{Z}^{+}, \\
\left(x(s)-\sqrt{\theta}, y(s)-\frac{1+a c}{c}, z(s)+\frac{\sqrt{\theta}}{c}\right)^{T}=\xi(s), \quad s \in[-\tau, 0],
\end{array}\right.
$$

where time delays $\tau_{i}(t) \in[-\tau, 0](i=1,2,3)$ with the scalar $\tau$ being positive. $X(t-\tau(t))=$ $\left(X_{1}\left(t-\tau_{1}(t)\right), X_{2}\left(t-\tau_{2}(t)\right), X_{3}\left(t-\tau_{3}(t)\right)\right)^{T}$. Impulsive time $t_{k}\left(k \in \mathbb{Z}^{+}\right)$is a positive number, satisfying $t_{1}<t_{2}<\cdots<t_{k}<t_{k+1}<\cdots$ with $\lim _{k \rightarrow \infty} t_{k}=+\infty$.

As pointed out in [8], $k_{i}(i=1,2,3)$ represents the feedback profit coefficient, which is also stochastic in a dynamic economic market. The randomness has the Markov property, for the feedback coefficient for the next moment is only related to that of the current 
moment. Therefore, in this paper, the authors consider the Markovian jumping delayed impulsive financial system as follows:

$$
\left\{\begin{array}{l}
\dot{X}(t)=-A X(t)+f(X(t))+K(r(t))(X-X(t-\tau(t))), \\
\quad t \geq t_{0}=0, t \neq t_{k}, k \in \mathbb{Z}^{+}, \\
X\left(t_{k}^{+}\right)=X\left(t_{k}\right)=B_{k} X\left(t_{k}^{-}\right), \quad t=t_{k}, k \in \mathbb{Z}^{+} \triangleq\{1,2, \ldots\}, \\
X(s)=\xi(s), \quad s \in[-\tau, 0]
\end{array}\right.
$$

or

$$
\left\{\begin{array}{l}
\dot{x}=z+(y-a) x+k_{1}(r(t))\left(x-x\left(t-\tau_{1}(t)\right)\right), \quad t \geq 0, t \neq t_{k}, k=1,2, \ldots, \\
\dot{y}=1-b y-x^{2}+k_{2}(r(t))\left(y-y\left(t-\tau_{2}(t)\right)\right), \quad t \geq 0, t \neq t_{k}, k=1,2, \ldots, \\
\dot{z}=-x-c z+k_{3}(r(t))\left(z-z\left(t-\tau_{3}(t)\right)\right), \quad t \geq 0, t \neq t_{k}, k=1,2, \ldots, \\
\left(x\left(t_{k}^{+}\right)-\sqrt{\theta}, y\left(t_{k}^{+}\right)-\frac{1+a c}{c}, z\left(t_{k}^{+}\right)+\frac{\sqrt{\theta}}{c}\right)^{T} \\
\quad=B_{k}\left(x\left(t_{k}^{-}\right)-\sqrt{\theta}, y\left(t_{k}^{-}\right)-\frac{1+a c}{c}, z\left(t_{k}^{-}\right)+\frac{\sqrt{\theta}}{c}\right)^{T}, \quad t=t_{k}, k \in \mathbb{Z}^{+}, \\
\left(x(s)-\sqrt{\theta}, y(s)-\frac{1+a c}{c}, z(s)+\frac{\sqrt{\theta}}{c}\right)^{T}=\xi(s), \quad s \in[-\tau, 0]
\end{array}\right.
$$

where $A$ and $f$ are defined in (2.4), and

$$
K(r(t))=\left(\begin{array}{ccc}
k_{1}(r(t)) & 0 & 0 \\
0 & k_{2}(r(t)) & 0 \\
0 & 0 & k_{3}(r(t))
\end{array}\right) .
$$

$(\Omega, \mathcal{F}, \mathbb{P})$ is a complete probability space with a natural filtration $\left\{\mathcal{F}_{t}\right\}_{t \geq 0}$. Let $S=\{1,2, \ldots$, $\left.n_{0}\right\}$ and the random form process $\{r(t):[0,+\infty) \rightarrow S\}$ be a homogeneous, finite-state Markovian process with right continuous trajectories with generator $\Pi=\left(\gamma_{i j}\right)_{n_{0} \times n_{0}}$ and transition probability from mode $i$ at time $t$ to mode $j$ at time $t+\delta, i, j \in S$,

$$
\mathbb{P}(r(t+\delta)=j \mid r(t)=i)=\left\{\begin{array}{l}
\gamma_{i j} \delta+o(\delta), \quad j \neq i, \\
1+\gamma_{i j} \delta+o(\delta), \quad j=i,
\end{array}\right.
$$

where $\gamma_{i j} \geq 0$ is the transition probability rate from $i$ to $j(j \neq i)$ and $\gamma_{i i}=-\sum_{j=1, j \neq i}^{n_{0}} \gamma_{i j}, \delta>0$ and $\lim _{\delta \rightarrow 0} o(\delta) / \delta=0$.

\section{Main results}

Before giving the main results of this paper, we need to present some assumptions and notations.

For simplicity, in this section, denote the norm $\|\cdot\|$ as follows:

$$
\|Y\|^{2}=Y^{T} Y, \quad \text { where } Y \in R^{3} .
$$

Suppose that time delays $\tau_{i}(t) \in[0, \tau], i=1,2,3$. Assume that there are two positive scalars $M_{1}, M_{2}$ such that

$$
0<M_{1} \leq\|X(s)\|^{2} \leq M_{2}, \quad \forall s \in[-\tau,+\infty) .
$$


In this paper, we assume that $X(t)$ is uniformly bounded on $[-\tau,+\infty)$, which implies that $\|X(t)\|^{2}$ is uniformly bounded on $[-\tau,+\infty)$, too. Thus, for any given $c_{\tau}>0$, there exists the corresponding $\delta_{c}>0$ such that

$$
\left|\|X(t)\|^{2}-\|X(t-\tau(t))\|^{2}\right|<c_{\tau} \quad \text { whenever } \tau \leq \delta_{c} .
$$

So, in this paper, we assume that

$$
\tau \leq \delta_{c}
$$

Remark 2 In the real financial market, some financial indicators, such as the interest rate, the investment demand, and the price index, are all percentages, and hence, the boundedness assumptions on these financial indicators are very natural, in line with the actual situation.

Theorem 3.1 Assume that $\sup _{k \in \mathbb{Z}^{+}}\left(t_{k}-t_{k-1}\right)<+\infty$. Assume, in addition, that there exist positive scalars $\varepsilon, \varsigma, \lambda$ such that

$$
\begin{aligned}
& \lambda_{\max } B_{k}^{T} B_{k}<e^{-(\varsigma+\lambda)\left(t_{k+1}-t_{k}\right)}, \quad k \in \mathbb{Z}^{+}, \\
& \lambda_{\max }\left(-A-A^{T}+2 K+\varepsilon^{-1} K^{2}+\varepsilon\left(1+\frac{c_{\tau}}{M_{1}}\right) I\right) \leq \varsigma-\lambda,
\end{aligned}
$$

where I represents the unit matrix with suitable dimension.

Then the following two conclusions hold simultaneously:

(a) The null solution of system (2.5) is globally exponentially stable with convergence rate $\frac{\lambda}{2}$

(b) The equilibrium point $Q_{1}$ with positive interest rate $\sqrt{\theta}$ for system (2.6) is globally exponentially stable with convergence rate $\frac{\lambda}{2}$.

Proof Firstly, combining (3.1)-(3.3) results in

$$
\left|\|X(t)\|^{2}-\|X(t-\tau(t))\|^{2}\right|<c_{\tau} \frac{\|X(t)\|^{2}}{M_{1}}
$$

which means

$$
\|X(t-\tau(t))\|^{2} \leq\left(1+\frac{c_{\tau}}{M_{1}}\right)\|X(t)\|^{2}, \quad \forall t \geq 0 .
$$

Next, it follows from (3.4) that there exists a positive number $q_{k}$ such that

$$
\lambda_{\max } B_{k}^{T} B_{k} \leq q_{k}<e^{-(\varsigma+\lambda)\left(t_{k+1}-t_{k}\right)}, \quad k \in \mathbb{Z}^{+} .
$$

Let $X$ be a solution of impulsive system (2.5), and consider the following Lyapunov function:

$$
V(t)=X^{T} X .
$$


Next, we claim that there exists a positive scalar

$$
M \geq \sup _{k \in \mathbb{Z}^{+}} e^{(\varsigma+\lambda)\left(t_{k}-t_{k-1}\right)}>1
$$

such that

$$
V(t) \leq M\|\xi\|_{\tau}^{2} e^{-\lambda\left(t-t_{0}\right)}, \quad t \in\left[t_{k-1}, t_{k}\right), k \in \mathbb{Z}^{+} .
$$

We may firstly prove that

$$
V(t) \leq M\|\xi\|_{\tau}^{2} e^{-\lambda\left(t-t_{0}\right)}, \quad t \in\left[t_{0}, t_{1}\right) .
$$

To prove (3.10), we only need

$$
V(t) \leq M\|\xi\|_{\tau}^{2} e^{-\lambda\left(t_{1}-t_{0}\right)}, \quad t \in\left[t_{0}, t_{1}\right) .
$$

Obviously, (3.11) holds in $t=t_{0}$ because the definition of $M$ deduces that

$$
M \geq e^{(\varsigma+\lambda)\left(t_{1}-t_{0}\right)}>e^{\lambda\left(t_{1}-t_{0}\right)} \quad \Rightarrow \quad M e^{-\lambda\left(t_{1}-t_{0}\right)}>1,
$$

and hence

$$
V\left(t_{0}\right)=\left\|X\left(t_{0}\right)\right\|^{2} \leq\|\xi\|_{\tau}^{2}<M\|\xi\|_{\tau}^{2} e^{-\lambda\left(t_{1}-t_{0}\right)} .
$$

Next, we assume (3.11) does not hold for $t \in\left[t_{0}, t_{1}\right)$. Then there is $\bar{t} \in\left(t_{0}, t_{1}\right)$ such that

$$
V(\bar{t})>M\|\xi\|_{\tau}^{2} e^{-\lambda\left(t_{1}-t_{0}\right)} .
$$

It is not difficult to obtain

$$
V(\bar{t})>M\|\xi\|_{\tau}^{2} e^{-\lambda\left(t_{1}-t_{0}\right)} \geq\|\xi\|_{\tau}^{2} e^{\zeta\left(t_{1}-t_{0}\right)}>\|\xi\|_{\tau}^{2} \geq V\left(t_{0}+s\right)=V(s), \quad s \in[-\tau, 0],
$$

which implies that there exists $t^{*} \in\left(t_{0}, \bar{t}\right)$ such that

$$
V\left(t^{*}\right)=M\|\xi\|_{\tau}^{2} e^{-\lambda\left(t_{1}-t_{0}\right)}, \quad \text { and } \quad V(t) \leq M\|\xi\|_{\tau}^{2} e^{-\lambda\left(t_{1}-t_{0}\right)}, \quad t \in\left[t_{0}, t^{*}\right]
$$

Besides,

$$
V\left(t^{*}\right)=M\|\xi\|_{\tau}^{2} e^{-\lambda\left(t_{1}-t_{0}\right)}>\|\xi\|_{\tau}^{2} \geq\left\|X\left(t_{0}\right)\right\|^{2}=V\left(t_{0}\right)
$$

Thus, there is $t^{* *} \in\left[t_{0}, t^{*}\right)$ such that

$$
V\left(t^{* *}\right)=\|\xi\|_{\tau}^{2}
$$

Let $D^{+}$be upper right derivative (Dini derivative) such that

$$
\begin{aligned}
& D^{+} V(t) \leq \lambda_{\max }\left(-A-A^{T}+2 K+\varepsilon^{-1} K^{2}+\varepsilon\left(1+\frac{c_{\tau}}{M_{1}}\right) I\right) V(t) \leq(\varsigma-\lambda) V(t), \\
& t \in\left[t^{* *}, t^{*}\right] \subset\left[t_{0}, t_{1}\right)
\end{aligned}
$$


Then the definition of $M$ yields

$$
\begin{aligned}
V\left(t^{*}\right) & \leq V\left(t^{* *}\right) e^{(\varsigma-\lambda)\left(t^{*}-t^{* *}\right)}<V\left(t^{* *}\right) e^{\zeta\left(t^{*}-t^{* *}\right)} \\
& =\|\xi\|_{\tau}^{2} e^{\varsigma\left(t^{*}-t^{* *}\right)} \leq M\|\xi\|_{\tau}^{2} e^{-\lambda\left(t_{1}-t_{0}\right)}=V\left(t^{*}\right) .
\end{aligned}
$$

This is an obvious contradiction. And then both (3.11) and (3.10) are proved.

Suppose that (3.17) holds for $k=1,2, \ldots, m$. That is,

$$
V(t) \leq M\|\xi\|_{\tau}^{2} e^{-\lambda\left(t-t_{0}\right)}, \quad t \in\left[t_{k-1}, t_{k}\right), k=1,2, \ldots, m .
$$

Below, we shall prove

$$
V(t) \leq M\|\xi\|_{\tau}^{2} e^{-\lambda\left(t-t_{0}\right)}, \quad t \in\left[t_{m}, t_{m+1}\right)
$$

Similarly, we only need to prove

$$
V(t) \leq M\|\xi\|_{\tau}^{2} e^{-\lambda\left(t_{m+1}-t_{0}\right)}, \quad t \in\left[t_{m}, t_{m+1}\right) .
$$

At first, we claim

$$
V\left(t_{m}\right)<M\|\xi\|_{\tau}^{2} e^{-\lambda\left(t_{m+1}-t_{0}\right)} .
$$

In fact,

$$
\begin{aligned}
V\left(t_{m}\right) & \leq \lambda_{\max } B_{m}^{T} B_{m}\left\|X\left(t_{m}^{-}\right)\right\|^{2} \\
& \leq q_{m} M\|\xi\|_{\tau}^{2} e^{-\lambda\left(t_{m}-t_{0}\right)} \\
& <e^{-(\varsigma+\lambda)\left(t_{m+1}-t_{m}\right)} M\|\xi\|_{\tau}^{2} e^{-\lambda\left(t_{m}-t_{0}\right)} \\
& \leq M\|\xi\|_{\tau}^{2} e^{-\lambda\left(t_{m+1}-t_{0}\right)}
\end{aligned}
$$

Thus, if (3.20) does not hold, we know that there is $\tilde{t} \in\left(t_{m}, t_{m+1}\right)$ such that

$$
V(\tilde{t})>M\|\xi\|_{\tau}^{2} e^{-\lambda\left(t_{m+1}-t_{0}\right)} .
$$

Then there must exist $t_{*} \in\left(t_{m}, t_{m+1}\right)$ such that

$$
V\left(t_{*}\right)=M\|\xi\|_{\tau}^{2} e^{-\lambda\left(t_{m+1}-t_{0}\right)}, \quad \text { and } \quad V(t) \leq M\|\xi\|_{\tau}^{2} e^{-\lambda\left(t_{m+1}-t_{0}\right)}, \quad \forall t \in\left[t_{m}, t_{*}\right]
$$

It follows from (3.22) that

$$
V\left(t_{m}\right) \leq q_{m} M\|\xi\|_{\tau}^{2} e^{-\lambda\left(t_{m}-t_{0}\right)}<M\|\xi\|_{\tau}^{2} e^{-\lambda\left(t_{m+1}-t_{0}\right)},
$$

which implies that there must be $t_{* *} \in\left[t_{m}, t_{*}\right)$ such that

$$
V\left(t_{* *}\right)=q_{m} M\|\xi\|_{\tau}^{2} e^{-\lambda\left(t_{m}-t_{0}\right)} .
$$


Similarly,

$$
\begin{aligned}
& D^{+} V(t) \leq \lambda_{\max }\left(-A-A^{T}+2 K+\varepsilon^{-1} K^{2}+\varepsilon\left(1+\frac{c_{\tau}}{M_{1}}\right) I\right) V(t) \leq(\varsigma-\lambda) V(t), \\
& \quad t \in\left[t_{* *}, t_{*}\right] \subset\left[t_{m}, t_{m+1}\right),
\end{aligned}
$$

we have

$$
\begin{aligned}
V\left(t_{*}\right) & \leq V\left(t_{* *}\right) e^{(\varsigma-\lambda)\left(t_{*}-t_{* *}\right)} \\
& \leq e^{-(\varsigma+\lambda)\left(t_{m+1}-t_{m}\right)} M\|\xi\|_{\tau}^{2} e^{-\lambda\left(t_{m}-t_{0}\right)} e^{(\varsigma-\lambda)\left(t_{m+1}-t_{m}\right)} \\
& <e^{-\zeta\left(t_{m+1}-t_{m}\right)} M\|\xi\|_{\tau}^{2} e^{-\lambda\left(t_{m}-t_{0}\right)} e^{(\varsigma-\lambda)\left(t_{m+1}-t_{m}\right)}=V\left(t_{*}\right) .
\end{aligned}
$$

This is an obvious contradiction. And then both (3.20) and (3.19) are proved. Finally, it is not difficult to derive from condition (3.4)

$$
\|X(t)\|^{2}=V(t) \leq M\|\xi\|_{\tau}^{2} e^{-\lambda\left(t-t_{0}\right)}, \quad t \in\left[t_{k-1}, t_{k}\right], k \in \mathbb{Z}^{+},
$$

which implies the completeness of the proof.

Similar to the proof of Theorem 3.1, we can select the Lyapunov function $\mathcal{V}(t)=$ $X^{T}(t) P_{r} X(t)$, deriving directly the following theorem for the case of Markovian jumping systems.

Theorem 3.2 Assume that $\sup _{k \in \mathbb{Z}^{+}}\left(t_{k}-t_{k-1}\right)<+\infty$. Assume, in addition, that there are a sequence of positive diagonal definite matrices $P_{r}=\operatorname{diag}\left(p_{r 1}, p_{r 2}, p_{r 3}\right)(r \in S)$ with $p_{r 1}=p_{r 2}$ and positive scalars $\varepsilon, \varsigma, \lambda$ such that

$$
\begin{aligned}
& \lambda_{\max } B_{k}^{T} B_{k}<e^{-(\varsigma+\lambda)\left(t_{k+1}-t_{k}\right)}, \quad k \in \mathbb{Z}^{+}, \\
& \frac{\max _{r \in S} \lambda_{\max }\left(-P_{r} A-A^{T} P_{r}+2 K_{r} P_{r}+\varepsilon^{-1} K_{r}^{2} P_{r}^{2}+\varepsilon\left(1+\frac{c_{\tau}}{M_{1}}\right) I+\sum_{r \in S} \gamma_{r j} P_{j}\right)}{\min _{r \in S} \lambda_{\min } P_{r}} \\
& \quad \leq \varsigma-\lambda,
\end{aligned}
$$

where I represents the unit matrix with suitable dimension.

Then the following two conclusions hold simultaneously:

(a) The null solution of system (2.7) is stochastically globally exponentially stable with convergence rate $\frac{\lambda}{2}$;

(b) The equilibrium point $Q_{1}$ with positive interest rate $\sqrt{\theta}$ for system (2.8) is stochastically globally exponentially stable with convergence rate $\frac{\lambda}{2}$.

Remark 3 Theorem 3.1 and Theorem 3.2 aim to employ the pulse control stabilizing chaotic system under closed economy. But if the open economy system is involved, the 
corresponding external inputs system can be considered as follows:

$$
\left\{\begin{array}{l}
\dot{X}(t)=-A X(t)+f(X(t))+K(X-X(t-\tau(t)))+u(t), \\
\quad t \geq t_{0}=0, t \neq t_{k}, k \in \mathbb{Z}^{+}, \\
X\left(t_{k}^{+}\right)=X\left(t_{k}\right)=B_{k} X\left(t_{k}^{-}\right), \quad t=t_{k}, k \in \mathbb{Z}^{+} \triangleq\{1,2, \ldots\} \\
X(s)=\xi(s), \quad s \in[-\tau, 0],
\end{array}\right.
$$

where $u(t)$ is the external input. Here, we may assume that there is a positive scalar $c_{0}>0$ such that

$$
\|u(t)\| \leq c_{0}\|X(t)\|
$$

Since the acquisition of parameters is often uncertain in real financial markets, the robust stability should be considered for the following system with parametric uncertainty:

$$
\left\{\begin{array}{l}
\dot{X}(t)=-(A+\Delta A(t)) X(t)+f(X(t))+K(X-X(t-\tau(t)))+u(t), \\
\quad t \geq t_{0}=0, t \neq t_{k}, k \in \mathbb{Z}^{+}, \\
X\left(t_{k}^{+}\right)=X\left(t_{k}\right)=B_{k} X\left(t_{k}^{-}\right), \quad t=t_{k}, k \in \mathbb{Z}^{+} \triangleq\{1,2, \ldots\}, \\
X(s)=\xi(s), \quad s \in[-\tau, 0],
\end{array}\right.
$$

where the parameter uncertainties are restricted as follows:

$$
A_{1} \leq \Delta A(t) \leq A_{2}
$$

or

$$
A+A_{1} \leq A+\Delta A(t) \leq A+A_{2},
$$

where $A_{1}$ and $A_{2}$ are known real constant matrices.

Theorem 3.3 Assume that $\sup _{k \in \mathbb{Z}^{+}}\left(t_{k}-t_{k-1}\right)<+\infty$ and the external input condition (3.29) holds. Assume, in addition, that there exist positive scalars $\varepsilon, \varsigma, \lambda$ with $\varsigma>\lambda$ such that

$$
\begin{aligned}
& 0<\lambda_{\max } B_{k}^{T} B_{k}<e^{-(\varsigma+\lambda)\left(t_{k+1}-t_{k}\right)}, \quad k \in \mathbb{Z}^{+}, \\
& \lambda_{\max }\left(-A-A_{1}-A^{T}-A_{1}^{T}\right)+\lambda_{\max }\left(2 K+I+c_{0}^{2} I+K\right)+\gamma e^{\lambda \tau} \lambda_{\max } K \leq \varsigma-\lambda,
\end{aligned}
$$

where $\gamma \geq \frac{1}{\lambda_{\max } B_{k}^{T} B_{k}}, k \in \mathbb{Z}^{+}$.

Then the null solution of the open economy system (3.30) is globally exponentially robust input-to-state stability with convergence rate $\frac{\lambda}{2}$.

Proof Let $D^{+}$be the upper right derivative (Dini derivative) along with system (3.30) such that

$$
\begin{aligned}
D^{+}\left(\|X(t)\|^{2}\right)= & X^{T}[-(A+\Delta A(t)) X(t)+f(X(t))+K(X-X(t-\tau(t)))+u(t)] \\
& +[-(A+\Delta A(t)) X(t)+f(X(t))+K(X-X(t-\tau(t)))+u(t)]^{T} X(t)
\end{aligned}
$$




$$
\begin{aligned}
\leq & \left(\lambda_{\max }\left(-A-A_{1}-A^{T}-A_{1}^{T}\right)+\lambda_{\max }\left(2 K+I+c_{0}^{2} I+K\right)\right)\|X(t)\|^{2} \\
& +\left(\lambda_{\max } K\right)\|X(t-\tau(t))\|^{2} .
\end{aligned}
$$

It follows from the assumption on $\gamma$ that there is a positive scalar $M>1$ such that

$$
\gamma e^{\lambda \tau}>M>\max \left\{e^{(\varsigma+\lambda)\left(t_{1}-t_{0}\right)}, e^{\lambda \tau}\right\}
$$

Next, we claim that

$$
\|X(t)\|^{2} \leq M\|\xi\|_{\tau}^{2} e^{-\lambda\left(t-t_{0}\right)}, \quad \forall t \in\left[t_{k-1}, t_{k}\right), k \in \mathbb{Z}^{+} .
$$

Indeed, employing mathematical induction will prove that (3.36) holds.

At first, we need to prove

$$
\|X(t)\|^{2} \leq M\|\xi\|_{\tau}^{2} e^{-\lambda\left(t-t_{0}\right)}, \quad \forall t \in\left[t_{0}, t_{1}\right)
$$

which implies that we only need to show

$$
\|X(t)\|^{2} \leq M\|\xi\|_{\tau}^{2} e^{-\lambda\left(t_{1}-t_{0}\right)}, \quad \forall t \in\left[t_{0}, t_{1}\right) .
$$

In fact, it is obvious that

$$
\left\|X\left(t_{0}\right)\right\|^{2} \leq\|\xi\|_{\tau}^{2}<M\|\xi\|_{\tau}^{2} e^{-\lambda\left(t_{1}-t_{0}\right)} .
$$

Thus, if (3.38) does not hold, there must exist some $t \in\left(t_{0}, t_{1}\right)$ such that $\|X(t)\|^{2}>$ $M\|\xi\|_{\tau}^{2} e^{-\lambda\left(t_{1}-t_{0}\right)}$, which implies that there is $t^{*} \in\left(t_{0}, t_{1}\right)$ satisfying

$$
\begin{aligned}
& \left\|X\left(t^{*}\right)\right\|^{2}=M\|\xi\|_{\tau}^{2} e^{-\lambda\left(t_{1}-t_{0}\right)}, \quad \text { and } \\
& \|X(t)\|^{2} \leq M\|\xi\|_{\tau}^{2} e^{-\lambda\left(t_{1}-t_{0}\right)}=\left\|X\left(t^{*}\right)\right\|^{2}, \quad \forall t \in\left[t_{0}, t^{*}\right)
\end{aligned}
$$

which together with (3.39) means that there is $t^{* *} \in\left[t_{0}, t^{*}\right)$ such that $\left\|X\left(t^{* *}\right)\right\|^{2}=\|\xi\|_{\tau}^{2}$ and

$$
\left\|X\left(t^{* *}\right)\right\|^{2} \leq\|X(t)\|^{2} \leq\left\|X\left(t^{*}\right)\right\|^{2}, \quad \forall t \in\left[t^{* *}, t^{*}\right] .
$$

On the other hand, it is obvious that

$$
\left\|X\left(t_{0}+s\right)\right\|^{2}=\|\xi\|^{2} \leq\|\xi\|_{\tau}^{2}<M\|\xi\|_{\tau}^{2} e^{-\lambda\left(t_{1}-t_{0}\right)}, \quad \forall s \in[-\tau, 0]
$$

which together with (3.40) implies

$$
\|X(t+s)\|^{2} \leq M\|\xi\|_{\tau}^{2} e^{-\lambda\left(t_{1}-t_{0}\right)}, \quad \forall s \in[-\tau, 0], t \in\left[t_{0}, t^{*}\right] .
$$

Besides, for any $s \in[-\tau, 0]$, we can conclude that

$$
\begin{aligned}
\|X(t+s)\|^{2} & \leq M\|\xi\|_{\tau}^{2} e^{-\lambda\left(t_{1}-t_{0}\right)} \\
& \leq \gamma e^{\lambda \tau}\left\|X\left(t^{* *}\right)\right\|^{2} \\
& \leq \gamma e^{\lambda \tau}\|X(t)\|^{2}, \quad \forall t \in\left[t^{* *}, t^{*}\right],
\end{aligned}
$$


which together with (3.34) and (3.33) implies that

$$
\begin{aligned}
D^{+}\left(\|X(t)\|^{2}\right) \leq & \left(\lambda_{\max }\left(-A-A_{1}-A^{T}-A_{1}^{T}\right)\right. \\
& \left.+\lambda_{\max }\left(2 K+I+c_{0}^{2} I+K\right)+\gamma e^{\lambda \tau} \lambda_{\max } K\right)\|X(t)\|^{2} \\
\leq & (\varsigma-\lambda)\|X(t)\|^{2}, \quad \forall t \in\left[t^{* *}, t^{*}\right],
\end{aligned}
$$

from which one can conclude

$$
\begin{aligned}
\left\|X\left(t^{*}\right)\right\|^{2} & \leq\left\|X\left(t^{* *}\right)\right\|^{2} e^{(\zeta-\lambda)\left(t^{*}-t^{* *}\right)} \\
& <M\|\xi\|_{\tau}^{2} e^{-\lambda\left(t_{1}-t_{0}\right)}=\left\|X\left(t^{*}\right)\right\|^{2} .
\end{aligned}
$$

This contradiction implies that (3.38) holds, and then (3.37) holds.

Next, we assume that (3.36) holds for $k=1,2, \ldots, m$, or

$$
\|X(t)\|^{2} \leq M\|\xi\|_{\tau}^{2} e^{-\lambda\left(t-t_{0}\right)}, \quad \forall t \in\left[t_{k-1}, t_{k}\right), k=1,2, \ldots, m .
$$

Below, we shall employ assumption (3.42) to conclude

$$
\|X(t)\|^{2} \leq M\|\xi\|_{\tau}^{2} e^{-\lambda\left(t-t_{0}\right)}, \quad \forall t \in\left[t_{m}, t_{m+1}\right) .
$$

It is obvious that

$$
\left\|X\left(t_{m}\right)\right\|^{2}<M\|\xi\|_{\tau}^{2} e^{-\lambda\left(t_{m}-t_{0}\right)} .
$$

Indeed, (3.42) yields

$$
\begin{aligned}
\left\|X\left(t_{m}\right)\right\|^{2} & =\left\|X\left(t_{m}^{+}\right)\right\|^{2} \\
& \leq\left(\lambda_{\max } B_{m}^{T} B_{m}\right) M\|\xi\|_{\tau}^{2} e^{-\lambda\left(t_{m}-t_{0}\right)} \\
& <e^{-\lambda\left(t_{m+1}-t_{m}\right)} M\|\xi\|_{\tau}^{2} e^{-\lambda\left(t_{m}-t_{0}\right)} \\
& <M\|\xi\|_{\tau}^{2} e^{-\lambda\left(t_{m}-t_{0}\right)} .
\end{aligned}
$$

Suppose that (3.44) is not true. Define $t_{b}=\inf \left\{t \in\left[t_{m}, t_{m+1}\right): V(t)>M\|\xi\|_{\tau}^{2} e^{-\lambda\left(t-t_{0}\right)}\right\}$. Then the continuity of $V(t)$ on $\left[t_{m}, t_{m+1}\right)$ derives

$$
\left\|X\left(t_{b}\right)\right\|^{2}=M\|\xi\|_{\tau}^{2} e^{-\lambda\left(t_{b}-t_{0}\right)} \quad \text { and } \quad\|X(t)\|^{2} \leq M\|\xi\|_{\tau}^{2} e^{-\lambda\left(t-t_{0}\right)}, \quad \forall t \in\left[t_{m}, t_{b}\right] .
$$

And (3.45) yields $t_{m}<t_{b}<t_{m+1}$. On the other hand,

$$
\begin{aligned}
\left\|X\left(t_{m}\right)\right\|^{2} & =\left\|X\left(t_{m}^{+}\right)\right\|^{2} \\
& \leq\left(\lambda_{\max } B_{m}^{T} B_{m}\right) e^{\lambda\left(t_{m+1}-t_{m}\right)} M\|\xi\|_{\tau}^{2} e^{-\lambda\left(t_{b}-t_{0}\right)} \\
& <M\|\xi\|_{\tau}^{2} e^{-\lambda\left(t_{b}-t_{0}\right)} .
\end{aligned}
$$

So the continuity of $\|X(t)\|^{2}$ on $\left[t_{m}, t_{m+1}\right)$ yields that there must be some $t \in\left[t_{m}, t_{b}\right)$ such that $\|X(t)\|^{2}=\left(\lambda_{\max } B_{m}^{T} B_{m}\right) e^{\lambda\left(t_{m+1}-t_{m}\right)} M\|\xi\|_{\tau}^{2} e^{-\lambda\left(t_{b}-t_{0}\right)}$. Define $t_{*}=\sup \left\{t \in\left[t_{m}, t_{b}\right):\|X(t)\|^{2}=\right.$ 
$\left.\left(\lambda_{\max } B_{m}^{T} B_{m}\right) e^{\lambda\left(t_{m+1}-t_{m}\right)} M\|\xi\|_{\tau}^{2} e^{-\lambda\left(t_{b}-t_{0}\right)}\right\}$, and then we can see from (3.47) and the definition of $t_{a}$ that

$$
\begin{aligned}
& \left\|X\left(t_{a}\right)\right\|^{2}=\left(\lambda_{\max } B_{m}^{T} B_{m}\right) e^{\lambda\left(t_{m+1}-t_{m}\right)} M\|\xi\|_{\tau}^{2} e^{-\lambda\left(t_{b}-t_{0}\right)} \quad \text { and } \\
& \left\|X\left(t_{a}\right)\right\|^{2} \leq\|X(t)\|^{2}, \quad \forall t \in\left[t_{a}, t_{b}\right] .
\end{aligned}
$$

Now, for $t \in\left[t_{a}, t_{b}\right]$ and $s \in[-\tau, 0]$, then $t+s \in\left[-\tau, t_{b}\right]$.

In the case of $t+s \in\left[-\tau, t_{m}\right),(3.42)$ yields

$$
\begin{aligned}
\|X(t+s)\|^{2} & \leq M\|\xi\|_{\tau}^{2} e^{-\lambda\left(t+s-t_{0}\right)} \\
& \leq M\|\xi\|_{\tau}^{2} e^{\lambda\left(t_{m+1}-t_{m}\right)} e^{-\lambda\left(t_{b}-t_{0}\right)} e^{\lambda \tau}, \quad \forall t \in\left[t_{a}, t_{b}\right] \subset\left[t_{m}, t_{m+1}\right) .
\end{aligned}
$$

In the case of $t+s \in\left[t_{m}, t_{b}\right]$, (3.46) yields

$$
\begin{aligned}
\|X(t+s)\|^{2} & \leq M\|\xi\|_{\tau}^{2} e^{-\lambda\left(t+s-t_{0}\right)} \\
& \leq M\|\xi\|_{\tau}^{2} e^{\lambda\left(t_{m+1}-t_{m}\right)} e^{-\lambda\left(t_{b}-t_{0}\right)} e^{\lambda \tau}, \quad \forall t \in\left[t_{a}, t_{b}\right] \subset\left[t_{m}, t_{m+1}\right) .
\end{aligned}
$$

Hence, combining (3.48)-(3.50) gives

$$
\begin{aligned}
\|X(t+s)\|^{2} & \leq M\|\xi\|_{\tau}^{2} e^{\lambda\left(t_{m+1}-t_{m}\right)} e^{-\lambda\left(t_{b}-t_{0}\right)} e^{\lambda \tau} \\
& \leq \gamma e^{\lambda \tau}\left\|X\left(t_{a}\right)\right\|^{2} \\
& \leq \gamma e^{\lambda \tau}\|X(t)\|^{2}, \quad \forall t \in\left[t_{a}, t_{b}\right], s \in[-\tau, 0],
\end{aligned}
$$

which together with (3.34) and (3.33) implies

$$
\begin{aligned}
D^{+}\left(\|X(t)\|^{2}\right) \leq & \left(\lambda_{\max }\left(-A-A_{1}-A^{T}-A_{1}^{T}\right)\right. \\
& \left.+\lambda_{\max }\left(2 K+I+c_{0}^{2} I+K\right)+\gamma e^{\lambda \tau} \lambda_{\max } K\right)\|X(t)\|^{2} \\
\leq & (\varsigma-\lambda)\|X(t)\|^{2}, \quad \forall t \in\left[t_{a}, t_{b}\right] .
\end{aligned}
$$

Similarly, we have

$$
\begin{aligned}
\left\|X\left(t_{b}\right)\right\|^{2} & \leq\left(\lambda_{\max } B_{m}^{T} B_{m}\right) e^{\lambda\left(t_{m+1}-t_{m}\right)} M\|\xi\|_{\tau}^{2} e^{-\lambda\left(t_{b}-t_{0}\right)} e^{(\varsigma-\lambda)\left(t_{b}-t_{a}\right)} \\
& \leq e^{-\varsigma\left(t_{m+1}-t_{m}\right)} M\|\xi\|_{\tau}^{2} e^{-\lambda\left(t_{b}-t_{0}\right)} e^{(\varsigma-\lambda)\left(t_{m+1}-t_{m}\right)} \\
& <M\|\xi\|_{\tau}^{2} e^{-\lambda\left(t_{b}-t_{0}\right)}=\left\|X\left(t_{b}\right)\right\|^{2}
\end{aligned}
$$

This contradiction verifies (3.43), and hence mathematical induction demonstrates claim (3.36), which derives

$$
\|X(t)\| \leq \sqrt{M}\|\xi\|_{\tau} e^{-\frac{\lambda}{2}\left(t-t_{0}\right)}, \quad \forall t \in\left[t_{k-1}, t_{k}\right), k \in \mathbb{Z}^{+} .
$$

Similar to the proof of [ 8 , Theorem 2], we can actually conclude from the above inequality that

$$
\|X(t)\| \leq \sqrt{M}\|\xi\|_{\tau} e^{-\frac{\lambda}{2}\left(t-t_{0}\right)}, \quad \forall t \in\left[t_{k-1}, t_{k}\right], k \in \mathbb{Z}^{+} .
$$


Therefore, the null solution of system (3.30) is globally exponentially robust input-tostate stability with convergence rate $\frac{\lambda}{2}$.

Remark 4 In [29, Theorem 3.1], there is assumption condition (ii) as follows:

(ii) $E \mathcal{L} V(t, \varphi) \leq-\lambda E V(t, \varphi(0))$, whenever $e^{\eta \theta} E V(t+\theta, \varphi(\theta))<q E V(t, \varphi(0))$ for all $\alpha \leq \theta \leq$ 0 , where $\varphi \in B L_{\mathcal{F}_{t}}^{p}$.

However, the conditions of our Theorems 3.1-3.3 reveal that our main matrix $-A$ is not necessarily negative definite. Actually, numerical examples show that the maximum eigenvalue of $-A$ is a positive number (see Examples 1-3), which is one of the main difficulties to be overcome in this paper.

Remark 5 Theorem 3.3 does not require the boundedness hypothesis of the state variable $X$, which is a perceptible improvement on Theorem 3.1. Of course, the state variable $X$ is an economic index, which itself is a bounded percentage. Besides, the boundedness assumption makes condition (3.5) of Theorem 3.1 simpler than the corresponding condition (3.33) of Theorem 3.3.

Remark 6 If the external input $u(t) \equiv 0$, the open economy system (3.30) becomes the closed economy system with parametric uncertainty as follows:

$$
\left\{\begin{array}{l}
\dot{X}(t)=-(A+\Delta A(t)) X(t)+f(X(t))+K(X-X(t-\tau(t))), \\
\quad t \geq t_{0}=0, t \neq t_{k}, k \in \mathbb{Z}^{+}, \\
X\left(t_{k}^{+}\right)=X\left(t_{k}\right)=B_{k} X\left(t_{k}^{-}\right), \quad t=t_{k}, k \in \mathbb{Z}^{+} \triangleq\{1,2, \ldots\} \\
X(s)=\xi(s), \quad s \in[-\tau, 0],
\end{array}\right.
$$

which implies that Theorem 3.3 includes the robust stability result of the closed economy system (3.52), and restriction condition (3.29) is naturally deleted.

Corollary 3.4 Assume that $\sup _{k \in \mathbb{Z}^{+}}\left(t_{k}-t_{k-1}\right)<+\infty$. Assume, in addition, that there exist positive scalars $\varepsilon, \varsigma, \lambda$ with $\varsigma>\lambda$ such that

$$
\begin{aligned}
& 0<\lambda_{\max } B_{k}^{T} B_{k}<e^{-(\varsigma+\lambda)\left(t_{k+1}-t_{k}\right)}, \quad k \in \mathbb{Z}^{+}, \\
& \lambda_{\max }\left(-A-A_{1}-A^{T}-A_{1}^{T}\right)+\lambda_{\max }(2 K+I+K)+\gamma e^{\lambda \tau} \lambda_{\max } K \leq \varsigma-\lambda,
\end{aligned}
$$

where $\gamma \geq \frac{1}{\lambda_{\max } B_{k}^{T} B_{k}}, k \in \mathbb{Z}^{+}$.

Then the null solution of system (3.52) is globally exponentially robust stability with convergence rate $\frac{\lambda}{2}$.

Remark 7 As far as we know, even for closed economic systems, Corollary 3.4 is a new criterion of robust stability.

Remark 8 If restriction condition (3.29) is abolished in Theorem 3.3, the regional control may be necessary for stabilization of the financial system. And so the following regional 
control systems have to be investigated for the open economy:

$$
\left\{\begin{array}{l}
\frac{\partial Z}{\partial t}=D \Delta Z-A Z(t, x)+f(Z(t, x))+K(Z-Z(t-\tau(t), x))+u(t, x), \\
\quad t \geq t_{0}=0, t \neq t_{k}, k=1,2, \ldots, \\
Z\left(t_{k}^{+}, x\right)=B_{k} Z\left(t_{k}^{-}, x\right), \quad t=t_{k}, k=1,2, \ldots \\
Z(s, x)=\xi(s, x), \quad(s, x) \in[-\tau, 0] \times \Omega, \\
\frac{\partial Z_{i}}{\partial v}=0, \quad x \in \partial \Omega, t \geq 0, i=1,2,3
\end{array}\right.
$$

or

$$
\left\{\begin{aligned}
& \frac{\partial Z(t, x)}{\partial t}= B \Delta Z(t, x)-A Z(t, x)+f(Z(t, x)) \\
&+K(r(t))(Z(t, x)-Z(t-\tau(t), x))+u(t, x), \quad t \geq 0, \\
& \frac{\partial Z}{\partial v}=0, \quad x \in \partial \Omega, \\
& Z(s, x)=\phi(s, x), \quad(s, x) \in[-\tau, 0] \times \Omega .
\end{aligned}\right.
$$

\section{Numerical examples}

Example 1 In system (2.5) or (2.6), let $a=0.9, b=0.2, c=0.2463$, and one gets

$$
A=\left(\begin{array}{ccc}
-4.0601 & -0.0893 & -1.0000 \\
0.1787 & 0.2000 & 0 \\
1.0000 & 0 & 0.2463
\end{array}\right) \text {. }
$$

In addition, let $\varsigma=9.3, \lambda=0.7, t_{k+1}-t_{k} \equiv 0.05$,

$$
\begin{aligned}
B_{k} & =\left(\begin{array}{ccc}
\frac{1}{18 k^{3}} & 0 & 0 \\
0 & \frac{1}{16 k^{5}} & 0 \\
0 & 0 & 0.0010
\end{array}\right), \quad k=1,2, \ldots . \\
K & =\left(\begin{array}{ccc}
0.07 & 0 & 0 \\
0 & 0.03 & 0 \\
0 & 0 & 0.01
\end{array}\right) .
\end{aligned}
$$

Let $\varepsilon=0.1, M_{1}=c_{\tau}=0.0001$, one can compute and obtain

$$
\begin{aligned}
& \lambda_{\max } B_{k}^{T} B_{k} \leq \lambda_{\max } B_{1}^{T} B_{1}<0.6065=e^{-(\varsigma+\lambda)\left(t_{k+1}-t_{k}\right)}, \\
& \varsigma-\lambda=8.6>\lambda_{\max }\left(-A-A^{T}+2 K+\varepsilon^{-1} K^{2}+\varepsilon\left(1+\frac{c_{\tau}}{M_{1}}\right) I\right),
\end{aligned}
$$

which implies that conditions (3.4) and (3.5) are satisfied. And hence, Theorem 3.1 yields that the equilibrium point $Q_{1}$ with positive interest rate $8.93 \%$ for financial system (2.6) is globally exponentially stable with convergence rate 0.35 .

Example 2 In system (2.7) or (2.8), let $a=0.9, b=0.2, c=0.2463$, and one gets

$$
A=\left(\begin{array}{ccc}
-4.0601 & -0.0893 & -1.0000 \\
0.1787 & 0.2000 & 0 \\
1.0000 & 0 & 0.2463
\end{array}\right) \text {. }
$$


In addition, let $q_{k} \equiv 0.6, \varsigma=9.3, \lambda=0.7, t_{k+1}-t_{k} \equiv 0.05$,

$$
B_{k}=\left(\begin{array}{ccc}
\frac{1}{18 k^{3}} & 0 & 0 \\
0 & \frac{1}{16 k^{5}} & 0 \\
0 & 0 & 0.0010
\end{array}\right), \quad k=1,2, \ldots
$$

Let $S=\{1,2\}$, and the transition rates matrix and feedback coefficient matrix $\Pi$ and feedback gain coefficient matrix $K_{r}$ are given as follows:

$$
\begin{aligned}
\Pi & =\left(\begin{array}{cc}
-0.013 & 0.013 \\
0.015 & -0.015
\end{array}\right), \quad K_{1}=\left(\begin{array}{ccc}
0.011 & 0 & 0 \\
0 & 0.015 & 0 \\
0 & 0 & 0.012
\end{array}\right), \\
K_{2} & =\left(\begin{array}{ccc}
0.012 & 0 & 0 \\
0 & 0.016 & 0 \\
0 & 0 & 0.013
\end{array}\right) . \\
P_{1} & =\left(\begin{array}{ccc}
1.005 & 0 & 0 \\
0 & 1.005 & 0 \\
0 & 0 & 0.9987
\end{array}\right), \quad P_{2}=\left(\begin{array}{ccc}
0.9993 & 0 & 0 \\
0 & 0.9993 & 0 \\
0 & 0 & 1.002
\end{array}\right) .
\end{aligned}
$$

Let $\varepsilon=0.1, M_{1}=c_{\tau}=0.0001$, one can compute and obtain

$$
\begin{aligned}
& \lambda_{\max } B_{k}^{T} B_{k} \leq \lambda_{\max } B_{1}^{T} B_{1}<0.6065=e^{-(\varsigma+\lambda)\left(t_{k+1}-t_{k}\right)}, \\
& \varsigma-\lambda=8.6>\frac{\max _{r \in S} \lambda_{\max }\left(-P_{r} A-A^{T} P_{r}+2 K_{r} P_{r}+\varepsilon^{-1} K_{r}^{2} P_{r}^{2}+\varepsilon\left(1+\frac{c_{\tau}}{M_{1}}\right) I+\sum_{r \in S} \gamma_{r j} P_{j}\right)}{\min _{r \in S} \lambda_{\min } P_{r}},
\end{aligned}
$$

which implies that conditions (3.26) and (3.27) are satisfied. And hence, Theorem 3.2 yields that the equilibrium point $Q_{1}$ with positive interest rate $8.93 \%$ for financial system (2.8) is stochastically globally exponentially stable with convergence rate 0.35 .

Remark 9 As pointed out in Remark 1, Theorem 3.1 and Theorem 3.2 may be the important conclusions of the first paper on the financial systems with time delays, although the time delays may be small.

Example 3 In system (3.30), let $a=0.9, b=0.2, c=0.2463$, and one gets

$$
A=\left(\begin{array}{ccc}
-4.0601 & -0.0893 & -1.0000 \\
0.1787 & 0.2000 & 0 \\
1.0000 & 0 & 0.2463
\end{array}\right) .
$$

In addition, let

$$
A_{1}=\left(\begin{array}{ccc}
0.00011 & 0 & 0 \\
0 & 0.00012 & 0 \\
0 & 0 & 0.00013
\end{array}\right)
$$


and $\varsigma=9.8, \lambda=0.2, t_{k+1}-t_{k} \equiv 0.05$,

$$
\begin{aligned}
B_{k} & =\left(\begin{array}{ccc}
\frac{1}{18 k^{3}} & 0 & 0 \\
0 & \frac{1}{16 k^{5}} & 0 \\
0 & 0 & 0.0010
\end{array}\right), \quad k=1,2, \ldots \\
K & =\left(\begin{array}{ccc}
0.0007 & 0 & 0 \\
0 & 0.0003 & 0 \\
0 & 0 & 0.0001
\end{array}\right) .
\end{aligned}
$$

Let $\tau=0.5, c_{0}=0.03, \gamma=260$, one can compute and obtain

$$
\begin{aligned}
\lambda_{\max } B_{k}^{T} B_{k} \leq \lambda_{\max } B_{1}^{T} B_{1}=0.0039<0.6065=e^{-(\varsigma+\lambda)\left(t_{k+1}-t_{k}\right)}, \\
\varsigma-\lambda=9.6>9.4237 \\
\quad=\lambda_{\max }\left(-A-A_{1}-A^{T}-A_{1}^{T}\right)+\lambda_{\max }\left(2 K+I+c_{0}^{2} I+K\right)+\gamma e^{\lambda \tau} \lambda_{\max } K,
\end{aligned}
$$

which implies that conditions (3.32) and (3.33) are satisfied. And hence, Theorem 3.3 yields that the open economy system (3.30) is globally exponentially robust input-to-state stable with convergence rate 0.1 , where the interest rate is $8.93 \%>0$ when the economy system reaches stability.

Remark 10 In Example 3, the upper bound of time delay is $\tau=0.5$, which is not small. This fully illustrates the effectiveness and feasibility of Theorem 3.3.

\section{Conclusions and further considerations}

In this paper, using some mathematical analysis techniques and Lyapunov function methods, we have derived the globally exponential stability criteria for the closed economy system and the open economy system. Because the interest rate obtained in Chinese financial market is usually positive, the global asymptotic stabilization of the positive interest rate equilibrium under impulse control studied in this paper has certain theoretical significance for Chinese economic management departments. Particularly, our Theorem 3.3 involves the robust input-to-state stabilization of the open economy system under impulse control while China's economy is an open economy. Moreover, global exponential stability implies deleting chaos of complex economy system.

As pointed out in [8] and [30], under Lipschitz conditions ensuring the unique existence of the solution of the reaction-diffusion system for any given initial value, Ruofeng Rao, Shouming Zhong, and Zhilin Pu deduced the boundedness conclusion [30, Theorem 3.3] and the stability criterion [30, Theorem 3.4], in which the following formula was derived:

$$
\lambda_{1}\left(B\left(0, R_{0}\right)\right)=\left\{\begin{array}{l}
\frac{\beta_{0}^{2}\left(\frac{\pi^{m}}{m !}\right) \frac{2}{2 m}}{\left[\operatorname{mes}\left(B\left(0, R_{0}\right)\right)\right] \frac{2}{2 m}}=\frac{\beta_{0}^{2}\left(\frac{\pi^{\frac{n}{2}}}{\left(\frac{n}{2}\right) !} \frac{2}{n}\right.}{\left[\operatorname{mes}\left(B\left(0, R_{0}\right)\right)\right] \frac{2}{n}}, \quad n=2 m, \\
\frac{\beta_{0}^{2} \frac{\left[2(2 \pi)^{m}\right] 2 m+1}{(2 m+1) ! !}}{\left[\operatorname{mes}\left(B\left(0, R_{0}\right)\right)\right] \frac{2}{2 m+1}}=\frac{\beta_{0}^{2} \frac{\left[2(2 \pi)^{\frac{n-1}{2}}\right.}{n ! !} \frac{2}{n}}{\left[\operatorname{mes}\left(B\left(0, R_{0}\right)\right)\right]^{\frac{2}{n}}}, \quad n=2 m+1 .
\end{array}\right.
$$

This has actually proven the following conclusion. 
Theorem 5.1 ([8, Theorem 3]) If $f_{i}, \tilde{f}_{i}, \sigma_{i j}$, $\hat{\sigma}_{i j}$ are Lipschitz continuous with $f_{i}(0)=\tilde{f}_{i}(0)=$ $\sigma_{i j}(0)=\hat{\sigma}_{i j}(0)=0$, then there must exist a series of spherical regions $B\left(0, R_{0}\right) \subset R^{n}$ with $R_{0}$ moderately small such that the following fuzzy system (5.2) is globally stochastically exponentially stable in the pth moment, where $\Upsilon=B\left(0, R_{0}\right)$ in (5.2).

$$
\left\{\begin{aligned}
d u_{i}(t, x)= & q_{i} \operatorname{div} \nabla u_{i}(t, x) d t-\sum_{r=1}^{r_{*}} \varrho_{r}(\hat{\omega}(t))\left[a_{i r} u_{i}(t, x)-\sum_{j=1}^{n} b_{i j r} f_{j}\left(v_{j}(t, x)\right)\right. \\
& \left.-\sum_{j=1}^{n} c_{i j r} f_{j}\left(v_{j}(t-\tau(t), x)\right)-\sum_{j=1}^{n} h_{i j r} \int_{t-\rho(t)}^{t} f_{j}\left(v_{j}(s, x)\right) d s\right] d t \\
& +\sum_{j=1}^{n} \sigma_{i j}(t, u(t, x), v(t-\tau(t), x)) d w_{j}(t), \quad t \geq 0, x \in \Upsilon \\
d v_{i}(t, x)= & \tilde{q}_{i} \operatorname{div} \nabla v_{i}(t, x) d t-\sum_{r=1}^{r_{*}} \varrho_{r}(\hat{\omega}(t))\left[\tilde{a}_{i r} v_{i}(t, x)\right. \\
& -\sum_{j=1}^{n} \tilde{b}_{i j r} \tilde{f}_{j}\left(u_{j}(t, x)\right)-\sum_{j=1}^{n} \tilde{c}_{i j} \tilde{f}_{j}\left(u_{j}(t-\hat{\tau}(t), x)\right) \\
& \left.-\sum_{j=1}^{n} \tilde{h}_{i j r} \int_{t-\tilde{\rho}(t)}^{t} \tilde{f}_{j}\left(u_{j}(s, x)\right) d s\right] d t \\
& +\sum_{j=1}^{n} \hat{\sigma}_{i j}(t, v(t, x), u(t-\hat{\tau}(t), x),) d \tilde{w}_{j}(t), \quad t \geq 0, x \in \Upsilon \\
u_{i}(t, x)= & \zeta_{i}(t, x), \quad v_{i}(t, x)=\varpi_{i}(t, x), \quad \forall(s, x) \in[-\tau, 0] \times \Upsilon \\
\partial_{\nu} u(t, x)= & 0=\partial_{\nu} v(t, x), \quad \forall(t, x) \in[0,+\infty] \times \partial \Upsilon .
\end{aligned}\right.
$$

Due to $\lambda_{1}(\Omega) \geq \lambda_{1}\left(B\left(0, R_{0}\right)\right)$ when $\Omega \subset B\left(0, R_{0}\right)$, we can actually generalize Theorem 5.1 and [30, Theorem 3.4] from the spherical region $B\left(0, R_{0}\right)$ to a more general region $\Omega \subset \mathbb{R}^{n}$, where $\Omega$ is a bounded domain of $\mathbb{R}^{n}$ with smooth boundary ([6, Theorem 6.3]). Moreover, it follows from [6, Theorem 6.2] and [6, Theorem 6.3] that

Theorem 5.2 If $f_{i}, \tilde{f}_{i}, \sigma_{i j}, \hat{\sigma}_{i j}$ are Lipschitz continuous with $f_{i}(0)=\tilde{f}_{i}(0)=\sigma_{i j}(0)=\hat{\sigma}_{i j}(0)=0$, then there must exist a series of domains $\Omega \subset B\left(a, R_{0}\right) \subset R^{n}$ with $R_{0}$ moderately small such that the following fuzzy system (5.2) is globally stochastically exponentially stable in the pth moment, where $\Upsilon=\Omega$ in (5.2).

How to derive the concise criterion for the open economy systems (3.54), (3.55), and (3.30), similar to that of Theorem 5.2, is an interesting problem.

\section{Funding}

The research is supported by the National Natural Science Foundation of China (Nos. 61533006), the China National College Students Innovation and Entrepreneurship Training Program at Chengdu Normal University in 2018 and 2019 (No. 201814389083; No. 201914389037), the Scientific Fund of the Education Department of Sichuan Province in China (No. 18ZA0082), the Application basic research project of science and Technology Department of Sichuan Province and the Major scientific research projects of Chengdu Normal University in 2019 (CS19ZDZ01).

\section{Competing interests}

The authors declare no conflict of interest.

\section{Authors' contributions}

Both authors read and approved the final manuscript.

\section{Author details}

${ }^{1}$ Department of Mathematics, Chengdu Normal University, Chengdu, China. ${ }^{2}$ College of Mathematics, University of Electronic Science and Technology of China, Chengdu, China. ${ }^{3}$ Institute of Financial Mathematics, Chengdu Normal University, Chengdu, China.

\section{Publisher's Note}

Springer Nature remains neutral with regard to jurisdictional claims in published maps and institutional affiliations. 


\section{References}

1. Cheng, S.: Complicated science and management. In: Article Collection of Beijing Xiangshan Conference, vol. 1. Science Press, Beijing (1998) (in Chinese)

2. Huang, D., Li, H.: Theory and Method of Nonlinear Economics. Sichuan University Press, Chengdu (1993)

3. Ma, J., Chen, Y.: Study for the bifurcation topological structure and the global complicated character of a kind of nonlinear finance system (I). Appl. Math. Mech. 11, 1240-1251 (2001)

4. Ma, J., Chen, Y.: Study for the bifurcation topological structure and the global complicated character of a kind of nonlinear finance system (II). Appl. Math. Mech. 12, 1375-1382 (2001)

5. Chen, W.: Dynamics and control of a financial system with time-delayed feedbacks. Chaos Solitons Fractals 37(4), 1198-1207 (2008)

6. Rao, R., Zhong, S.: Input-to-state stability and no-inputs stabilization of delayed feedback chaotic financial system involved in open and closed economy. Discrete Contin. Dyn. Syst., Ser. S 1-19 (2020). https://doi.org/10.3934/dcdss.2020280

7. Zhao, X., Li, Z., Li, S.: Synchronization of a chaotic finance system. Appl. Math. Comput. 217,6031-6039 (2011)

8. Rao, R.: Global stability of a Markovian jumping chaotic financial system with partially unknown transition rates under impulsive control involved in the positive interest rate. Mathematics 7(7), 579 (2019) https://doi.org/10.3390/math7070579

9. Huang, T., Li, C., Liu, X.: Synchronization of chaotic systems with delay using intermittent linear state feedback. Chaos $18,033122(2008)$

10. Zhang, Y., Wang, Q.: Comment on "Synchronization of chaotic systems with delay using intermittent linear state feedback". Chaos 18, 048101 (2008)

11. Long, X., Gong, S.: New results on stability of Nicholson's blowflies equation with multiple pairs of time-varying delays. Appl. Math. Lett. 100, Article 106027 (2020). https://doi.org/10.1016/j.aml.2019.106027

12. Huang, C., Zhang, H., Huang, L.: Almost periodicity analysis for a delayed Nicholson's blowflies model with nonlinear density-dependent mortality term. Commun. Pure Appl. Anal. 18(6), 3337-3349 (2019)

13. Huang, C., Nie, X., Zhao, X., Song, Q., Cao, J.: Novel bifurcation results for a delayed fractional-order quaternion-valued neural network. Neural Netw. 117, 67-93 (2019)

14. Chen, T., Huang, L., Yu, P., Huang, W.: Bifurcation of limit cycles at infinity in piecewise polynomial systems. Nonlinear Anal., Real World Appl. 41, 82-106 (2018)

15. Huang, C., Yang, Z., Yi, T., Zou, X.: On the basins of attraction for a class of delay differential equations with non-monotone bistable nonlinearities. J. Differ. Equ. 256, 2101-2114 (2014)

16. Rao, R., Zhong, S., Wang, X.: Stochastic stability criteria with LMI conditions for Markovian jumping impulsive BAM neural networks with mode-dependent time-varying delays and nonlinear reaction-diffusion. Commun. Nonlinear Sci. Numer. Simul. 19, 258-273 (2014)

17. Huang, C., Peng, C., Chen, X., Wen, F.: Dynamics analysis of a class of delayed economic model. Abstr. Appl. Anal. 2013 Article ID 962738 (2013)

18. Huang, C., Su, R., Cao, J., Xiao, S.: Asymptotically stable high-order neutral cellular neural networks with proportional delays and D operators. Math. Comput. Simul. (2019) In press, corrected proof, Available online 10 June 2019

19. Li, X., Song, S.: Research on synchronization of chaotic delayed neural networks with stochastic perturbation using impulsive control method. Commun. Nonlinear Sci. Numer. Simul. 19(10), 3892-3900 (2014)

20. Li, X., Zhu, Q., O'Regan, D.: pth moment exponential stability of impulsive stochastic functional differential equations and application to control problems of NNs. J. Franklin Inst. 351, 4435-4456 (2014)

21. Rao, R., Hang, J., Zhong, S.: Global exponential stability of reaction-diffusion BAM neural networks. J. Jilin Univ. Sci. Ed. 50, 1086-1090 (2012) (In Chinese)

22. Li, X., Yang, X., Huang, T.: Persistence of delayed cooperative models: impulsive control method. Appl. Math. Comput. $342,130-146(2019)$

23. Song, Q., Yu, Q., Zhao, Z., Liu, Y., Fuad, E.: Boundedness and global robust stability analysis of delayed complex-valued neural networks with interval parameter uncertainties. Neural Netw. 103, 55-62 (2018)

24. Li, X., Shen, J., Rakkiyappan, R.: Persistent impulsive effects on stability of functional differential equations with finite or infinite delay. Appl. Math. Comput. 329, 14-22 (2018)

25. Song, Q., Yu, Q., Zhao, Z., Liu, Y., Fuad, E.: Dynamics of complex-valued neural networks with variable coefficients and proportional delays. Neurocomputing 275, 2762-2768 (2018)

26. Li, X., Ho, D., Cao, J.: Finite-time stability and settling-time estimation of nonlinear impulsive systems. Automatica 99 361-368 (2019)

27. Rao, R.: Delay-dependent exponential stability for nonlinear reaction-diffusion uncertain Cohen-Grossberg neural networks with partially known transition rates via Hardy-Poincare inequality. Chin. Ann. Math., Ser. B 35, 575-598 (2014)

28. Gao, Q., Ma, J.: Chaos and Hopf bifurcation of a finance system. Nonlinear Dyn. 58, 209 (2009). https://doi.org/10.1007/s11071-009-9472-5

29. Li, X., Fu, X.: Stability analysis of stochastic functional differential equations with infinite delay and its application to recurrent neural networks. J. Comput. Appl. Math. 234, 407-417 (2010)

30. Rao, R., Zhong, S., Pu, Z.: Fixed point and p-stability of TCS fuzzy impulsive reaction-diffusion dynamic neural networks with distributed delay via Laplacian semigroup. Neurocomputing 335, 170-184 (2019) 